\title{
LOCALIZED COHERENT STRUCTURES AND PATTERNS FORMATION IN COLLECTIVE MODELS OF BEAM MOTION
}

\author{
A. FEDOROVA, M. ZEITLIN \\ IPME, RAS, St. Petersburg, V.O. Bolshoj pr., 61, 199178, Russia \\ e-mail: zeitlin@math.ipme.ru \\ http://www.ipme.ru/zeitlin.htm; http://www.ipme.nw.ru/zeitlin.htm
}

\begin{abstract}
We present applications of variational - wavelet approach to three different models of nonlinear beam motions with underlying collective behaviour: Vlasov-MaxwellPoisson systems, envelope dynamics, beam-beam model. We have the representation for dynamical variables as a multiresolution (multiscales) expansion via high-localized nonlinear eigenmodes in the base of compactly supported wavelet bases. Numerical modelling demonstrates formation of coherent structures and stable patterns.
\end{abstract}

\section{Introduction}

In this paper we consider the applications of a new numerical-analytical technique which is based on the methods of local nonlinear harmonic analysis or wavelet analysis to three nonlinear beam/accelerator physics problems which can be characterized by collective type behaviour: some forms of Vlasov-Maxwell-Poisson equations[1], RMS envelope dynamics[2], the model of beam-beam interactions[3]. Such approach may be useful in all models in which it is possible and reasonable to reduce all complicated problems related with statistical distributions to the problems described by systems of nonlinear ordinary/partial differential equations with or without some (functional)constraints. Wavelet analysis is a relatively novel set of mathematical methods, which gives us the possibility to work with well-localized bases in functional spaces and gives the maximum sparse forms for the general type of operators (differential, integral, pseudodifferential) in such bases. Our approach is based on the variational-wavelet approach from [4]-[14], which allows us to consider polynomial and rational type of nonlinearities. The solution has the following multiscale/multiresolution decomposition via nonlinear highlocalized eigenmodes

$$
\begin{aligned}
u(t, x) & =\sum_{(i, j) \in Z^{2}} a_{i j} U^{i}(x) V^{j}(t), \\
V^{k}(t) & =V_{N}^{k, \text { slow }}(t)+\sum_{i \geq N} V_{i}^{k}\left(\omega_{i}^{1} t\right), \quad \omega_{i}^{1} \sim 2^{i}
\end{aligned}
$$




$$
U^{k}(x)=U_{M}^{k, \text { slow }}(x)+\sum_{j \geq M} U_{j}^{k}\left(\omega_{j}^{2} x\right), \quad \omega_{j}^{2} \sim 2^{j},
$$

which corresponds to the full multiresolution expansion in all underlying time/space scales ( $x$ are the generalized space coordinates or phase space coordinates, $t$ is time coordinate). Formula (11) gives us expansion into the slow part $u_{N, M}^{\text {slow }}$ and fast oscillating parts for arbitrary $\mathrm{N}, \mathrm{M}$. So, we may move from coarse scales of resolution to the finest one for obtaining more detailed information about our dynamical process. The first terms in the RHS of formulae (1)-(3) correspond on the global level of function space decomposition to resolution space and the second ones to detail space. In this way we give contribution to our full solution from each scale of resolution or each time/space scale or from each nonlinear eigenmode (Fig.1). The same is correct for the contribution to power spectral density (energy spectrum): we can take into account contributions from each level/scale of resolution. In all these models numerical modelling demonstrates the appearence of coherent high-localized structures and stable patterns formation. Starting in part 2 from Vlasov-Maxwell-Poisson equations, root-mean-square (RMS) envelope dynamics and beam-beam interaction model we consider in part 3 the approach based on variational-wavelet formulation. We give explicit representation for all dynamical variables in the base of compactly supported wavelets or nonlinear eigenmodes. Our solutions are parametrized by solutions of a number of reduced algebraical problems one from which is nonlinear with the same degree of nonlinearity and the rest are the linear problems which correspond to particular method of calculation of scalar products of functions from wavelet bases and their derivatives. In part 4 we consider numerical modelling based on our analytical approach.

\section{Collective models}

\subsection{Vlasov-Maxwell-Poisson Equations}

Analysis based on the non-linear Vlasov-Maxwell-Poisson equations leads to more clear understanding of the collective effects and nonlinear beam dynamics of high intensity beam propagation in periodic-focusing and uniformfocusing transport systems. We consider the following form of equations $([1],[2]$ for setup and designation):

$$
\begin{aligned}
& \left\{\frac{\partial}{\partial s}+p_{x} \frac{\partial}{\partial x}+p_{y} \frac{\partial}{\partial y}-\left[k_{x}(s) x+\frac{\partial \psi}{\partial x}\right] \frac{\partial}{\partial p_{x}}-\right. \\
& \left.\left[k_{y}(s) y+\frac{\partial \psi}{\partial y}\right] \frac{\partial}{\partial p_{y}}\right\} f_{b}\left(x, y, p_{x}, p_{y}, s\right)=0
\end{aligned}
$$

Capri-ICFA-Proc-2: submitted to World Scientific on October 26, 20182 


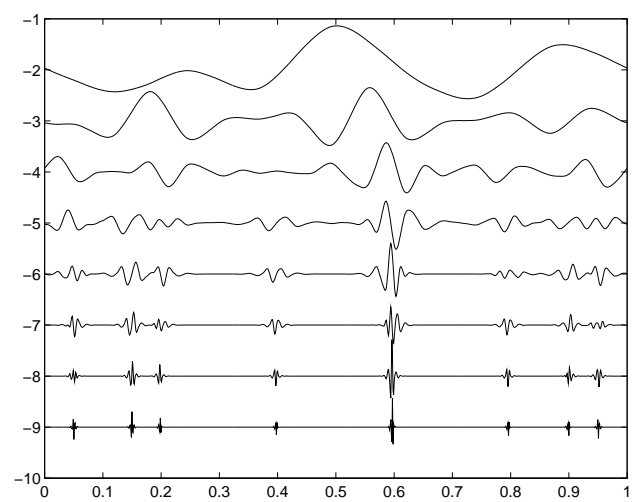

Figure 1. Multiscale/eigenmode decomposition.

$$
\begin{aligned}
& \left(\frac{\partial^{2}}{\partial x^{2}}+\frac{\partial^{2}}{\partial y^{2}}\right) \psi=-\frac{2 \pi K_{b}}{N_{b}} \int \mathrm{d} p_{x} \mathrm{~d} p_{y} f_{b}, \\
& \int \mathrm{d} x \mathrm{~d} y \mathrm{~d} p_{x} \mathrm{~d} p_{y} f_{b}=N_{b}
\end{aligned}
$$

The corresponding Hamiltonian for transverse single-particle motion is given by

$$
\begin{aligned}
& H\left(x, y, p_{x}, p_{y}, s\right)=\frac{1}{2}\left(p_{x}^{2}+p_{y}^{2}\right)+\frac{1}{2}\left[k_{x}(s) x^{2}\right. \\
& \left.+k_{y}(s) y^{2}\right]+H_{1}\left(x, y, p_{x}, p_{y}, s\right)+\psi(x, y, s),
\end{aligned}
$$

where $H_{1}$ is nonlinear (polynomial/rational) part of the full Hamiltonian. In case of Vlasov-Maxwell-Poisson system we may transform (4) into invariant form

$$
\frac{\partial f_{b}}{\partial s}+[f, H]=0
$$

\subsection{RMS Equations}

We consider an approach based on the second moments of the distribution functions for the calculation of evolution of RMS envelope of a beam. The RMS envelope equations are the most useful for analysis of the beam selfforces (space-charge) effects and also allow to consider both transverse and longitudinal dynamics of space-charge-dominated relativistic high-brightness

Capri-ICFA-Proc-2: submitted to World Scientific on October 26, $2018 \quad 3$ 
axisymmetric/asymmetric beams, which under short laser pulse-driven radiofrequency photoinjectors have fast transition from nonrelativistic to relativistic regime [2]. Analysis of halo growth in beams, appeared as result of bunch oscillations in the particle-core model, also are based on three-dimensional envelope equations [2]. We can consider the different forms of RMS envelope equations, which are not more than nonlinear differential equations with rational nonlinearities and variable coefficients from the formal point of view. Let $f\left(x_{1}, x_{2}\right)$ be the distribution function which gives full information about noninteracting ensemble of beam particles regarding to trace space or transverse phase coordinates $\left(x_{1}, x_{2}\right)$. Then we may extract the first nontrivial effects of collective dynamics from the second moments

$$
\sigma_{x_{i} x_{j}}^{2}=<x_{i} x_{j}>=\iint x_{i} x_{j} f\left(x_{i}, x_{j}\right) \mathrm{d} x_{i} \mathrm{~d} x_{j}
$$

RMS emittance ellipse is given by $\varepsilon_{x, r m s}^{2}=<x_{i}^{2}><x_{j}^{2}>-<x_{i} x_{j}>^{2}(i \neq$ $j$ ). Expressions for twiss parameters are also based on the second moments. We will consider the following particular cases of RMS envelope equations, which describe evolution of the moments (9) ([2] for full designation): for asymmetric beams we have the system of two envelope equations of the second order for $\sigma_{x_{1}}$ and $\sigma_{x_{2}}$ :

$$
\begin{aligned}
& \sigma_{x_{1}}^{\prime \prime}+\sigma_{x_{1}}^{\prime} \frac{\gamma^{\prime}}{\gamma}+\Omega_{x_{1}}^{2}\left(\frac{\gamma^{\prime}}{\gamma}\right)^{2} \sigma_{x_{1}}=I /\left(I_{0}\left(\sigma_{x_{1}}+\sigma_{x_{2}}\right) \gamma^{3}\right)+\varepsilon_{n x_{1}}^{2} / \sigma_{x_{1}}^{3} \gamma^{2}, \\
& \sigma_{x_{2}}^{\prime \prime}+\sigma_{x_{2}}^{\prime} \frac{\gamma^{\prime}}{\gamma}+\Omega_{x_{2}}^{2}\left(\frac{\gamma^{\prime}}{\gamma}\right)^{2} \sigma_{x_{2}}=I /\left(I_{0}\left(\sigma_{x_{1}}+\sigma_{x_{2}}\right) \gamma^{3}\right)+\varepsilon_{n x_{2}}^{2} / \sigma_{x_{2}}^{3} \gamma^{2}
\end{aligned}
$$

The envelope equation for an axisymmetric beam is a particular case of preceding equations. Also we have related Lawson's equation for evolution of the rms envelope in the paraxial limit, which governs evolution of cylindrical symmetric envelope under external linear focusing channel of strenght $K_{r}$ :

$$
\sigma^{\prime \prime}+\sigma^{\prime}\left(\frac{\gamma^{\prime}}{\beta^{2} \gamma}\right)+K_{r} \sigma=\frac{k_{s}}{\sigma \beta^{3} \gamma^{3}}+\frac{\varepsilon_{n}^{2}}{\sigma^{3} \beta^{2} \gamma^{2}},
$$

where $K_{r} \equiv-F_{r} / r \beta^{2} \gamma m c^{2}, \quad \beta \equiv \nu_{b} / c=\sqrt{1-\gamma^{-2}}$. According [2] we have the following form for envelope equations in the model of halo formation by bunch oscillations:

$$
\begin{aligned}
& \ddot{X}+k_{x}^{2}(s) X-\frac{3 K}{8} \frac{\xi_{x}}{Y Z}-\frac{\varepsilon_{x}^{2}}{X^{3}}=0 \\
& \ddot{Y}+k_{y}^{2}(s) Y-\frac{3 K}{8} \frac{\xi_{y}}{X Z}-\frac{\varepsilon_{y}^{2}}{Y^{3}}=0
\end{aligned}
$$




$$
\ddot{Z}+k_{z}^{2}(s) Z-\gamma^{2} \frac{3 K}{8} \frac{\xi_{z}}{X Y}-\frac{\varepsilon_{z}^{2}}{Z^{3}}=0
$$

where $\mathrm{X}(\mathrm{s}), \mathrm{Y}(\mathrm{s}), \mathrm{Z}(\mathrm{s})$ are bunch envelopes, $\xi_{x}, \xi_{y}, \xi_{z}=F(X, Y, Z)$.

After transformations to Cauchy form we can see that all these equations from the formal point of view are not more than ordinary differential equations with rational nonlinearities and variable coefficients Also, we may consider regimes in which $\gamma, \gamma^{\prime}$ are not fixed functions/constants but satisfy some additional differential constraints/equations, but this case does not change our general approach of the next part.

\subsection{Beam-beam modelling}

In A. Chao e.a. model [3] for simulation of beam-beam interaction the initial collective description by some sort of equation for distribution function $f(s, x, p)$

$$
\frac{\partial f}{\partial s}+p \frac{\partial f}{\partial x}-(k(s) x-F(x, s)) \frac{\partial f}{\partial p}=0
$$

is reduced to Fockker-Planck (FP) equation on the first stage and to very nontrivial dynamical system with complex behaviour,

$$
\begin{aligned}
& \frac{\mathrm{d}^{2} \sigma_{k}}{\mathrm{~d} s^{2}}+\Gamma_{k} \frac{\mathrm{d} \sigma_{k}}{\mathrm{~d} s}+F_{k} \sigma_{k}=\frac{1}{\beta_{k}^{2} a_{k}^{2} \sigma_{k}^{3}} \\
& \frac{\mathrm{d} a_{n}}{\mathrm{~d} s}=\Gamma_{k} a_{k}\left(1-a_{k}^{2} \sigma_{k}^{2}\right),
\end{aligned}
$$

which solution gives the parameters of enveloping gaussian anzatz for solution of FP equation, on the second stage. From the formal point of view equations (14) are particular case of system (12).

\section{$3 \quad$ Rational Dynamics}

After some anzatzes [15] our problems may be formulated as the systems of ordinary differential equations (cases 2.2 and 2.3 (system (14)) above)

$$
\begin{aligned}
& Q_{i}(x) \frac{\mathrm{d} x_{i}}{\mathrm{~d} t}=P_{i}(x, t), \quad x=\left(x_{1}, \ldots, x_{n}\right), \\
& i=1, \ldots, n, \quad \max _{i} \operatorname{deg} P_{i}=p, \quad \max _{i} \operatorname{deg} Q_{i}=q
\end{aligned}
$$

or a set of such systems (cases 2.1, 2.3 (full equation (13)) above) corresponding to each independent coordinate in phase space. They have the fixed

Capri-ICFA-Proc-2: submitted to World Scientific on October 26, 2018 
initial(or boundary) conditions $x_{i}(0)$, where $P_{i}, Q_{i}$ are not more than polynomial functions of dynamical variables $x_{j}$ and have arbitrary dependence of time. Because of time dilation we can consider only next time interval: $0 \leq t \leq 1$. Let us consider a set of functions

$$
\Phi_{i}(t)=x_{i} \frac{\mathrm{d}}{\mathrm{d} t}\left(Q_{i} y_{i}\right)+P_{i} y_{i}
$$

and a set of functionals

$$
F_{i}(x)=\int_{0}^{1} \Phi_{i}(t) d t-\left.Q_{i} x_{i} y_{i}\right|_{0} ^{1}
$$

where $y_{i}(t)\left(y_{i}(0)=0\right)$ are dual (variational) variables. It is obvious that the initial system and the system

$$
F_{i}(x)=0
$$

are equivalent. Of course, we consider such $Q_{i}(x)$ which do not lead to the singular problem with $Q_{i}(x)$, when $t=0$ or $t=1$, i.e. $Q_{i}(x(0)), Q_{i}(x(1)) \neq$ $\infty$.

Now we consider formal expansions for $x_{i}, y_{i}$ :

$$
x_{i}(t)=x_{i}(0)+\sum_{k} \lambda_{i}^{k} \varphi_{k}(t) \quad y_{j}(t)=\sum_{r} \eta_{j}^{r} \varphi_{r}(t)
$$

where $\varphi_{k}(t)$ are useful basis functions of some functional space $\left(L^{2}, L^{p}\right.$, Sobolev, etc) corresponding to concrete problem and because of initial conditions we need only $\varphi_{k}(0)=0, r=1, \ldots, N, \quad i=1, \ldots, n$,

$$
\lambda=\left\{\lambda_{i}\right\}=\left\{\lambda_{i}^{r}\right\}=\left(\lambda_{i}^{1}, \lambda_{i}^{2}, \ldots, \lambda_{i}^{N}\right),
$$

where the lower index i corresponds to expansion of dynamical variable with index i, i.e. $x_{i}$ and the upper index $r$ corresponds to the numbers of terms in the expansion of dynamical variables in the formal series. Then we put (19) into the functional equations (18) and as result we have the following reduced algebraical system of equations on the set of unknown coefficients $\lambda_{i}^{k}$ of expansions (19):

$$
L\left(Q_{i j}, \lambda, \alpha_{I}\right)=M\left(P_{i j}, \lambda, \beta_{J}\right),
$$

where operators L and M are algebraization of RHS and LHS of initial problem (15), where $\lambda$ (20) are unknowns of reduced system of algebraical equations (RSAE)(21).

$Q_{i j}$ are coefficients (with possible time dependence) of LHS of initial system of differential equations (15) and as consequence are coefficients of RSAE.

Capri-ICFA-Proc-2: submitted to World Scientific on October 26, $2018 \quad 6$ 
$P_{i j}$ are coefficients (with possible time dependence) of RHS of initial system of differential equations (15) and as consequence are coefficients of RSAE. $I=\left(i_{1}, \ldots, i_{q+2}\right), J=\left(j_{1}, \ldots, j_{p+1}\right)$ are multiindexes, by which are labelled $\alpha_{I}$ and $\beta_{I}$ are other coefficients of RSAE (21):

$$
\beta_{J}=\left\{\beta_{j_{1} \ldots j_{p+1}}\right\}=\int \prod_{1 \leq j_{k} \leq p+1} \varphi_{j_{k}},
$$

where $\mathrm{p}$ is the degree of polinomial operator $\mathrm{P}(15)$

$$
\alpha_{I}=\left\{\alpha_{i_{1}} \ldots \alpha_{i_{q+2}}\right\}=\sum_{i_{1}, \ldots, i_{q+2}} \int \varphi_{i_{1} \ldots} \dot{\varphi_{i_{s}} \ldots \varphi_{i_{q+2}}}
$$

where $\mathrm{q}$ is the degree of polynomial operator $\mathrm{Q}(15), i_{\ell}=(1, \ldots, q+2), \dot{\varphi_{i_{s}}}=$ $\mathrm{d} \varphi_{i_{s}} / \mathrm{d} t$.

Now, when we solve RSAE (21) and determine unknown coefficients from formal expansion (19) we therefore obtain the solution of our initial problem. It should be noted if we consider only truncated expansion (19) with $\mathrm{N}$ terms then we have from (21) the system of $N \times n$ algebraical equations with degree $\ell=\max \{p, q\}$ and the degree of this algebraical system coincides with degree of initial differential system. So, we have the solution of the initial nonlinear (rational) problem in the form

$$
x_{i}(t)=x_{i}(0)+\sum_{k=1}^{N} \lambda_{i}^{k} X_{k}(t),
$$

where coefficients $\lambda_{i}^{k}$ are roots of the corresponding reduced algebraical (polynomial) problem RSAE (21). Consequently, we have a parametrization of solution of initial problem by solution of reduced algebraical problem (21). The first main problem is a problem of computations of coefficients $\alpha_{I}$ (23), $\beta_{J}$ (22) of reduced algebraical system. These problems may be explicitly solved in wavelet approach [4]-[6]. The obtained solutions are given in the form (24), where $X_{k}(t)$ are basis functions and $\lambda_{k}^{i}$ are roots of reduced system of equations. In our case $X_{k}(t)$ are obtained via multiresolution expansions and represented by compactly supported wavelets and $\lambda_{k}^{i}$ are the roots of corresponding general polynomial system (21). Because affine group of translation and dilations is inside the approach, this method resembles the action of a microscope. We have contribution to final result from each scale of resolution from the whole infinite scale of spaces. More exactly, the closed subspace $V_{j}(j \in \mathbf{Z})$ corresponds to level $\mathrm{j}$ of resolution, or to scale $\mathrm{j}$. We consider a multiresolution analysis of $L^{2}\left(\mathbf{R}^{n}\right)$ (of course, we may consider any different 
functional space) which is a sequence of increasing closed subspaces $V_{j}$ :

$$
\ldots V_{-2} \subset V_{-1} \subset V_{0} \subset V_{1} \subset V_{2} \subset \ldots
$$

satisfying the following properties: let $W_{j}$ be the orthonormal complement of $V_{j}$ with respect to $V_{j+1}: V_{j+1}=V_{j} \oplus W_{j}$, then

$$
L^{2}(\mathbf{R})=\overline{V_{0} \bigoplus_{j=0}^{\infty} W_{j}}
$$

This functional space decomposition corresponds to exact nonlinear eigen mode decompositions (2),(3). It should be noted that such representations give the best possible localization properties in the corresponding (phase)space/time coordinates. In contrast with different approaches formulae (1)-(3) do not use perturbation technique or linearization procedures and represent dynamics via generalized nonlinear localized eigenmodes expansion. So, by using wavelet bases with their good (phase)space/time localization properties we can construct high-localized coherent structures in spatially-extended stochastic systems with collective behaviour.

\section{$4 \quad$ Modelling}

Resulting multiresolution/multiscale representations for solutions of equations from part 2 in the high-localized bases/eigenmodes are demonstrated on Fig. 2-Fig. 7. This modelling demonstrates the appearence of stable patterns formation from high-localized coherent structures. On Fig. 2, Fig. 3 we present contribution to the full expansion (1)-(3) from level 1 and level 4 of decomposition (25). Figures 4, 5 show the representations for full solutions, constructed from the first 6 eigenmodes (6 levels in formula (25)). Figures 6 , 7 show stable patterns formation based on high-localized coherent structures.

\section{Acknowledgments}

We would like to thank Professor Pisin Chen, Dr. Stefania Petracca and her team for nice hospitality, help and support during Capri ICFA Workshop.

Capri-ICFA-Proc-2: submitted to World Scientific on October 26, $2018 \quad \mathbf{8}$ 


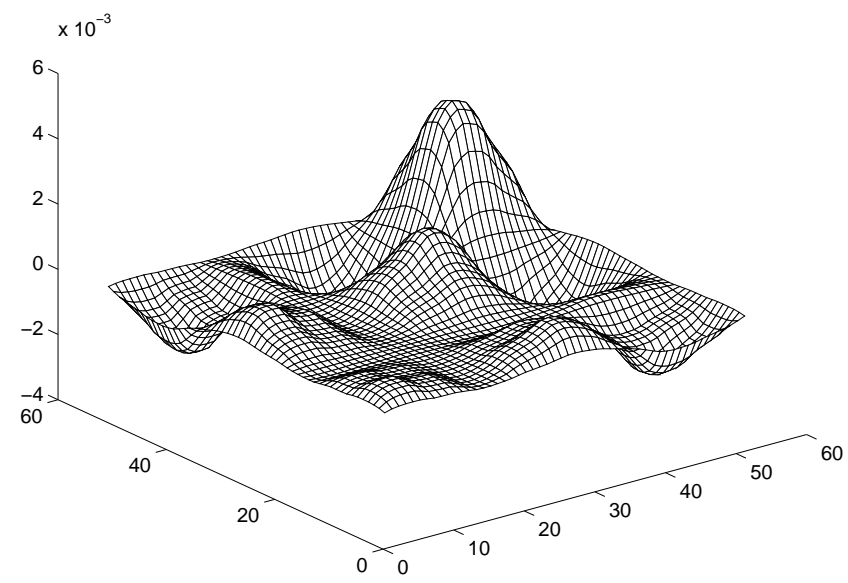

Figure 2. Eigenmode of level 1

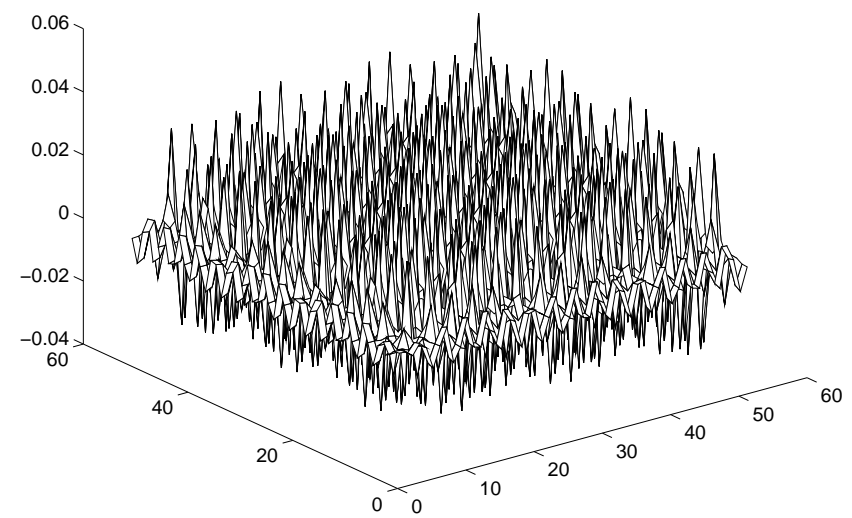

Figure 3. Eigenmode of level 4 


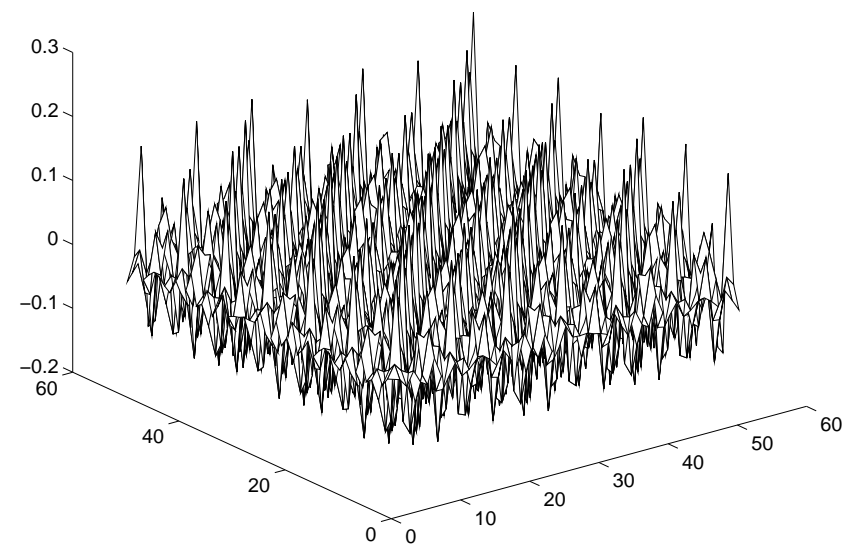

Figure 4. Appearence of coherent structure

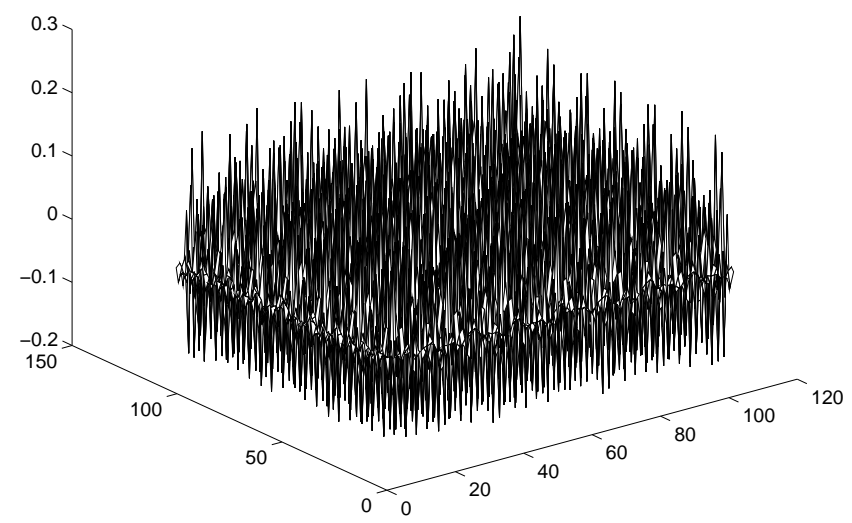

Figure 5. Six-eigenmodes decomposition 


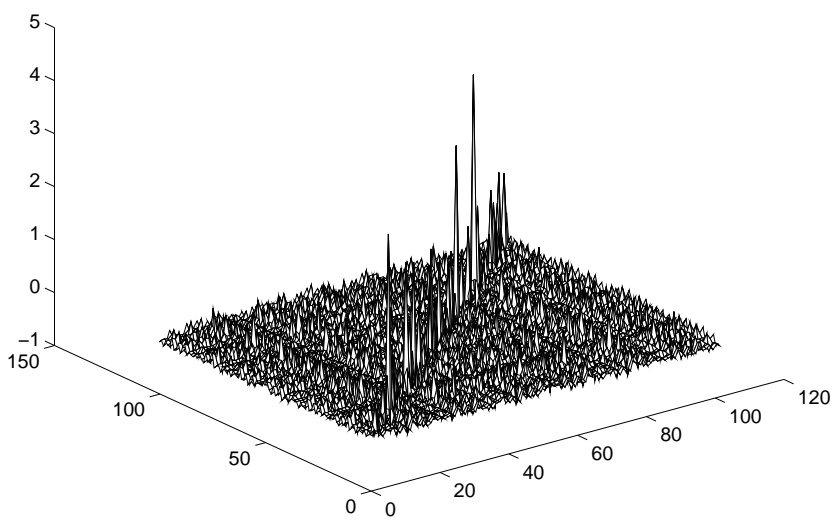

Figure 6. Stable pattern 1

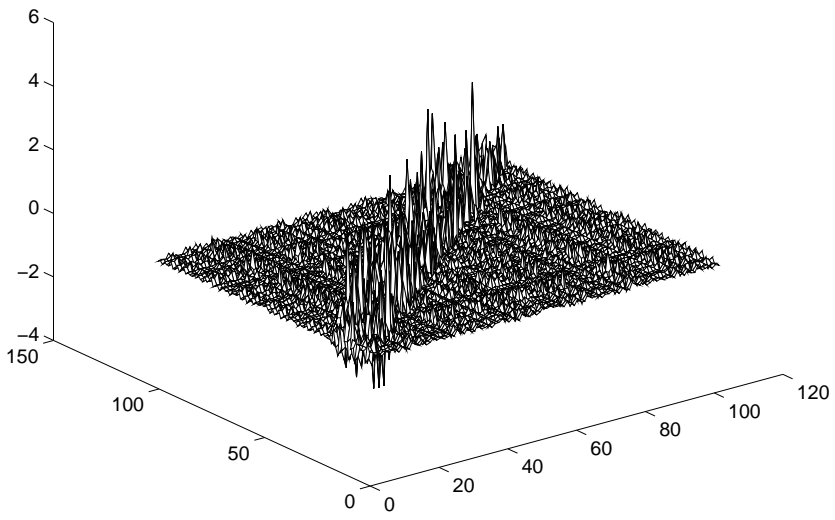

Figure 7. Stable pattern 2 


\section{References}

1. R. Davidson, H. Qin, P. Channel, PRSTAB, 2, 074401, 1999

2. J.B. Rosenzweig, Fundamentals of Beam Physics, e-version: http://www.physics.ucla.edu/class/99F/250Rosenzweig/notes/ L. Serafini and J.B. Rosenzweig, Phys. Rev. E 55, 7565, 1997. C. Allen, T. Wangler, papers in UCLA ICFA Proc., World Sci., 2000.

3. A. Chao, e.a., Los Alamos preprint, physics/0010055

4. A.N. Fedorova and M.G. Zeitlin, Math. and Comp. in Simulation, 46, 527 (1998).

5. A.N. Fedorova and M.G. Zeitlin, 'Wavelet Approach to Mechanical Problems. Symplectic Group, Symplectic Topology and Symplectic Scales', New Applications of Nonlinear and Chaotic Dynamics in Mechanics, 31, 101 (Kluwer, 1998).

6. A.N. Fedorova and M.G. Zeitlin, CP405, 87 (American Institute of Physics, 1997). Los Alamos preprint, physics/9710035.

7. A.N. Fedorova, M.G. Zeitlin and Z. Parsa, Proc. PAC97 2, 1502, 1505, 1508 (IEEE, 1998).

8. A.N. Fedorova, M.G. Zeitlin and Z. Parsa, Proc. EPAC98, 930, 933 (Institute of Physics, 1998).

9. A.N. Fedorova, M.G. Zeitlin and Z. Parsa, CP468, 48 (American Institute of Physics, 1999). Los Alamos preprint, physics/990262.

10. A.N. Fedorova, M.G. Zeitlin and Z. Parsa, CP468, 69 (American Institute of Physics, 1999). Los Alamos preprint, physics/990263.

11. A.N. Fedorova and M.G. Zeitlin, Proc. PAC99, 1614, 1617, 1620, 2900, 2903, 2906, 2909, 2912 (IEEE/APS, New York, 1999).

Los Alamos preprints: physics/9904039, 9904040, 9904041, 9904042, 9904043, 9904045, 9904046, 9904047.

12. A.N. Fedorova and M.G. Zeitlin, Proc. UCLA ICFA Workshop, in press, Los Alamos preprint: physics/0003095.

13. A.N. Fedorova and M.G. Zeitlin, Proc. EPAC00, 415, 872, 1101, 1190, 1339, 2325.

Los Alamos preprints: physics/0008045, 0008046, 0008047, 0008048, 0008049, 0008050

14. A.N. Fedorova, M.G. Zeitlin, Proc. LINAC00, 2 papers in press, Los Alamos preprints: physics/0008043, 0008200

15. A.N. Fedorova, M.G. Zeitlin, this Volume and in press.

Capri-ICFA-Proc-2: submitted to World Scientific on October 26, 201812 